\title{
Overexpression of SIOFP20 affects floral organ and pollen development
}

\author{
Shengen Zhou', Zongli Hu', Fenfen Li', Shibing Tian², Zhiguo Zhu', Anzhou Li and Guoping Chen
}

\begin{abstract}
The OVATE gene was initially identified in tomato and serves as a key regulator of fruit shape. There are 31 OFP members in the tomato genome. However, their roles in tomato growth and reproductive development are largely unknown. Here, we cloned the OFP transcription factor SIOFP20. Tomato plants overexpressing SIOFP2O displayed several phenotypic defects, including an altered floral architecture and fruit shape and reduced male fertility. SIOFP20 overexpression altered the expression levels of some brassinosteroid (BR)-associated genes, implying that SIOFP20 may play a negative role in the BR response, similar to its ortholog OsOFP19 in rice. Moreover, the transcript accumulation of gibberellin (GA)-related genes was significantly affected in the transgenic lines. SIOFP20 may play an important role in the crosstalk between BR and GA. The pollen germination assay suggested that the pollen germination rate of SIOFP20-OE plants was distinctly lower than that of WT plants. In addition, the tomato pollen-associated genes SICRK1, SIPMEI, LEPRK3, SIPRALF, and LAT52 were all suppressed in the transgenic lines. Our data imply that SIOFP2O may affect floral organ and pollen development by modulating BR and GA signaling in tomato.
\end{abstract}

\section{Introduction}

The reproductive development of most higher plants includes floral meristem determination, floral bud emergence, and fruit development and ripening, all of which result in seed formation and dispersal to guarantee offspring survival ${ }^{1}$. Numerous studies indicate that several gene families extensively participate in the transcriptional modulation of reproductive and developmental processes. For example, the microRNA156-targeted SPL/SBP box transcription factors control the processes of ovary and fruit development in tomato ${ }^{2}$. Silencing of SIDELLA, a GRAS transcription factor, induces facultative parthenocarpy in tomato fruits. A gain-of-function mutation in the MADS-box gene Sl-AGL11 exerts a great impact on the organization of flowers and fruit, especially on the transition of the sepals into a carpel-like fleshy organ, and on

\footnotetext{
Correspondence: Zongli Hu (huzongli71@163.com) or Guoping Chen (chenguoping@cqu.edu.cn)

'Laboratory of Molecular Biology of Tomato, Bioengineering College,

Chongqing University, Chongqing, People's Republic of China

${ }^{2}$ Institute of Vegetable Research, Chongqing Academy of Agricultural Sciences,

Chongqing, People's Republic of China
}

increases in sugar content and fruit softness ${ }^{3}$. The OVATE family proteins (OFPs) are plant-specific transcription factors. The OVATE gene was originally characterized as a key quantitative trait locus that contributes to the conversion of tomato fruit from round to pear shaped ${ }^{4}$. Amino acid sequence analysis shows that this gene encodes a hydrophilic protein including a putative bipartite nuclear localization signal and a C-terminal domain of $\sim 70$ amino acids referred to as the OVATE domain. Unlike other known transcription factor families, the OVATE proteins are a novel class of functional proteins ${ }^{5}$. OFPs have mainly been functionally characterized in Arabidopsis ${ }^{6-11}$ and rice ${ }^{12-15}$ and have been demonstrated to control diverse aspects of plant growth and development. Overexpression of AtOFP1 decreases the length of all aboveground organs, such as the hypocotyl, rosette leaf, floral organs and siliques, and chromatin immunoprecipitation analysis demonstrated that AtOFP1 directly regulates AtGA20ox1, encoding the key enzyme in GA biosynthesis ${ }^{7}$. Additionally, AtOFP1 may take part in DNA repair ${ }^{8}$. Moreover, plants overexpressing AtOFP2, 4 and 7 generate similar phenotypes to AtOFP1- 
overexpressing plants, such as kidney-shaped cotyledons and round and curled leaves ${ }^{7,10}$. Overexpression of OsOFP2 results in decreased plant height and an altered leaf morphology and seed shape in rice ${ }^{12}$. Overexpression of OsOFP1 in rice causes multiple phenotypes, including increased leaf angles, decreased plant height, and altered grain shape ${ }^{14}$. The interaction of MaOFP1 and MuMADS1 in banana plays an antagonistic role in ethylene-induced postharvest fruit ripening ${ }^{16}$. According to the most recent study, MuMADS1 and MaOFP1 control fruit quality in a tomato ovate mutant ${ }^{17}$. These results strongly support the notion that OFPs act as important regulatory factors in numerous processes in plant growth and development.

Studies on the mechanism of action of OFPs have shown that they function via interacting with different kinds of transcription factors, such as KNOX and BELL classes $^{6,13,18}$. The interaction networks between OFPs and TALE proteins have an important effect on plant developmental processes. The interaction of AtOFP1 and BLH3 has been shown to regulate the timing of conversion from the vegetative to reproductive stage in Arabidopsis $^{11}$. AtOFP4 has been proposed to interact with KNAT7 (Knotted1-Like Homeodomain Protein 7) to control the establishment of secondary cell walls by increasing the transcriptional repression activity of KNAT7 $7^{10}$. The interaction of AtOFP5 with KNAT3 and BLH1 inhibits the activity of BELL-KNOX TALE complexes to assure normal embryo sac development in Arabidopsis ${ }^{19}$. In Arabidopsis, the cell wall defect of the knat7 mutant can be partially restored by ectopic expression of GhKNL1, a homeodomain protein from cotton (Gossypium hirsutum); moreover, GhKNL1 can interact with GhOFP4, AtOFP1, and AtOFP4 ${ }^{20}$.

Hormone pathways extensively participate in the extraordinary plasticity of plant ontogeny. There are several classes of phytohormones, including auxins, brassinosteroids, and gibberellins, that play essential roles in the regulation of growth in general and of cell elongation in particular ${ }^{21}$. Previous reports suggest that OFPs affect plant developmental processes by modulating the brassinosteroid and gibberellin signaling pathways ${ }^{7,13-15}$. AtOFP1 controls cell elongation in part by regulating the mRNA accumulation of the gibberellin biosynthesis gene AtGA20ox $1^{7}$. OsOFP19, OSH1 and DLT form a complex that regulates the complicated balance between plant growth and development and brassinosteroid signaling ${ }^{15}$. Increased brassinosteroid signaling can induce the expression of OsOFP1 by OsBZR1 and promote protein stability by repressing OsGSK2, resulting in the activation of OsOFP1, which then bonds with DLT factors and regulates downstream genes such as gibberellin metabolism genes to control plant morphology and grain shape in rice $^{14}$. OsOFP8 acts as a positive regulator in the brassinosteroid signaling pathway by interacting with OsGKS2, which plays a negative role in the brassinosteroid signaling pathway ${ }^{13}$.

In view of the outstanding nutritive and commercial value of tomato (Solanum lycopersicum), it has been regarded as one of the most important vegetable crops. It is also a model organism for studying fleshy fruit development and ripening $^{22}$, compound leaf development, and floral system and plant architecture ${ }^{23}$. Genome-wide analysis of OFPs in tomato has been carried out, and there are 31 SlOFPs in the tomato genome ${ }^{24}$. Herein, we present the functional characterization of SLOFP2O (accession number: Solyc10g076180), a classic OFP family gene homologous to AtOFP1 and OsOFP19 in Arabidopsis and rice, respectively. It has been reported that $S I O F P 20$ is a suppressor of ovate in the modulation of fruit shape ${ }^{25}$. To investigate the role of SIOFP20 related to the development of vegetative and reproductive growth in tomato, the SlOFP2O gene was cloned and overexpressed in wild-type tomato, leading to pleiotropic phenotypes. In this study, we sought to reveal the impacts of SIOFP2O on reproductive development, including floral architecture and pollen development. Morphological, statistical, and molecular evidence is reported here to clarify the potential reasons for these phenotypes.

\section{Materials and methods}

\section{Plant materials and growth conditions}

Solanum lycopersicum Mill. cv. Ailsa Craig tomato plants were used as the wild-type (WT) in our research. WT and transgenic tomato plants were grown in a greenhouse under standard greenhouse conditions (16-h-day/8-h-night cycle, $25^{\circ} \mathrm{C} / 18^{\circ} \mathrm{C}$ day/night temperature). To determine the organ-specific expression pattern of SlOFP20, roots, stems, leaves, sepals, flowers, and fruits of different stages were sampled from WT tomato plants according to our previous report $^{26}$. The four-whorl floral organs (sepal, petal, stamen, and carpel) were also harvested. For 24-epibrassinolide (EBR) treatment, $10 \mu \mathrm{M}$ EBR and water (control) were sprayed on five-leaf-stage wild-type tomato plants. In addition, the third leaves from treated and untreated plants were harvested after $0,1,2,4,8,12$, and $24 \mathrm{~h}$. All samples used in this study were immediately frozen with liquid nitrogen and kept at $-80^{\circ} \mathrm{C}$.

\section{Sequence analysis and phylogenetic tree construction}

The protein sequence alignment of SIOFP20 and other OFP proteins was generated by using the DNAMAN 5.2.2 programs. The conserved OVATE domains were identified by using Scan Prosite (http://prosite.expasy.org/scanprosite/) to reveal the phylogenetic relationships of SlOFP20 with 17 OFP family proteins from Arabidopsis and rice. The maximum likelihood (ML) method was applied to construct a dendrogram with MEGA 6.06 software. The accuracy of this tree was ensured by the bootstrap test replicated 1000 times. 
The GenBank accession numbers of the proteins included in the tree were as follows: AtOFP1 (NP_195804), AtOFP2 (NP_180599), AtOFP4 (NP_172174), AtOFP5 (NP_193618), AtOFP6 (NP_680125), AtOFP7 (NP_179440), AtOFP8 (NP_197466), AtOFP13 (NM_196102), AtOFP15 (XM_565833), AtOFP16 (NP_180770), AtOFP18 (NP_566967), OsOFP1 (XP_015643684), and OsOFP19 (XP_015638848). The Tomato Solanaceae Genomics Network (SGN) unigene accession numbers were as follows: SlOFP5 (Solyc02g072030), SlOFP14 (Solyc06g082460), SlOFP15 (Solyc07g055240), SlOFP17 (Solyc09g018200), and SlOFP20 (Solyc10g076180).

\section{Vector construction and tomato transformation}

For the overexpression of SlOFP2O in WT tomato, the full-length sequence was amplified by high-fidelity PCR (Prime START mix DNA polymerase, Takara) with the SlOFP2O-F and SlOFP20-R primers (Supplementary Table $\mathrm{S} 1$ ), which were tailed with $X b a \mathrm{I}$ and $\mathrm{SacI}$ restriction sites, respectively, at their $5^{\prime}$ ends. A DNA-Tailing kit (Takara) was applied to tail the obtained PCR products, which were then linked with the pMD18-T vector (Takara). The correct pMD18-T-SIOFP20 plasmid was used as the template and was amplified with the primers SlOFP2O-F and SlOFP20-R. Then, the amplified products were inserted into the pBI121 vector. The resulting SIOFP20-OE vector was introduced into Agrobacterium LBA4404. Plant transformation was conducted following our previously published protocols ${ }^{27}$. Transgenic lines were screened on kanamycin medium and verified by genomic PCR using the NPTII-F and NPTII-R primers (Supplementary Table S1). The positive SlOFP20$\mathrm{OE}$ transgenic lines were retained and used for further studies.

\section{Gene expression analysis}

In this study, RNAiso Plus (Takara) was used to extract total RNA. One microgram of total RNA was reversetranscribed (M-MLV Reverse Transcriptase Kit, Promega). Transcript levels were evaluated by real-time quantitative PCR according to a method published previously $^{28}$. SlCAC (Solyc08g006960) was used as a reference gene ${ }^{29}$. The primer sequences are shown in Supplementary Table S1.

\section{Transactivation activity and yeast two-hybrid assay for SIOFP20}

Transactivation activity and yeast two-hybrid assays were performed according to our previous report ${ }^{26}$.

\section{Anatomic characterization and scanning electron microscopy}

For anatomic characterization, WT and OE3 flowers at anthesis were fixed with $70 \%$ ethanol/acetic acid/formaldehyde (18:1:1, v/v/v). Paraffin sections were prepared according to a previous report ${ }^{30}$. Transverse sections of the middle part of the flowers were observed with a microscope (OLYMPUS IX71) and photographed.

For SEM (scanning electron microscopy) analysis, fully open flowers from the WT and OE3 transgenic lines were collected and fixed with $2 \%$ glutaraldehyde. The samples were dehydrated in a gradient ethanol-water series. After vacuum drying, the stamens were separated and coated with gold for SEM observation and photography.

\section{Pollen germination and viability assays}

Pollen germination was performed as described previously $^{31}$. Briefly, WT and OE3 transgenic plant anthers at the anthesis stage were collected and transferred to germination solution. Released pollen was then germinated by incubation in the dark at $25^{\circ} \mathrm{C}$ for $3 \mathrm{~h}$ and were defined as germinated when the pollen tube was at least as long as the diameter of the pollen grain. Pollen germination was observed, and images were taken. A pollen viability assay was conducted as described previously ${ }^{32}$. Pollen grains of WT and OE3 plants at the anthesis stage were collected and soaked in a $0.1 \%$ 2,3,5-triphenyl-2 h-tetrazolium chloride (TTC) solution for $15 \mathrm{~min}$ to assess their activity. The experiments were repeated three times.

\section{Cross assay}

A cross assay was performed according to our previous report ${ }^{33}$. In brief, unopened flower buds from OE3 transgenic lines were emasculated. Mature pollen from the WT was transferred by brushing the WT anthers onto the stigmas of the OE3 transgenic lines.

\section{Seed germination assay}

Seeds from WT and SlOFP2O-OE transgenic tomato lines (T2) were used for germination assays. After surface sterilization, seeds ( $\sim 30$ seeds for each replicate) were sown onto MS medium and then germinated in the dark at $25^{\circ} \mathrm{C}$ for 10 days. Radicle emergence $>1 \mathrm{~mm}$ was regarded as seed germination. Seed germination rates were recorded daily. The experiments were repeated three times.

\section{Results}

SIOFP20 is a typical OVATE family protein

OVATE family proteins govern various developmental processes. To study the potential roles of OFP genes in tomato, we isolated an OFP gene (SlOFP20) from WT tomato on the basis of the sequence available in GenBank (accession no. XM_004248997). The SlOFP20 gene is intronless and has an open reading frame (ORF) of 966 nucleotides, which encodes a protein of 321 amino acid residues. Multiple sequence alignment analysis of SIOFP20 and other well-known OVATE family proteins 

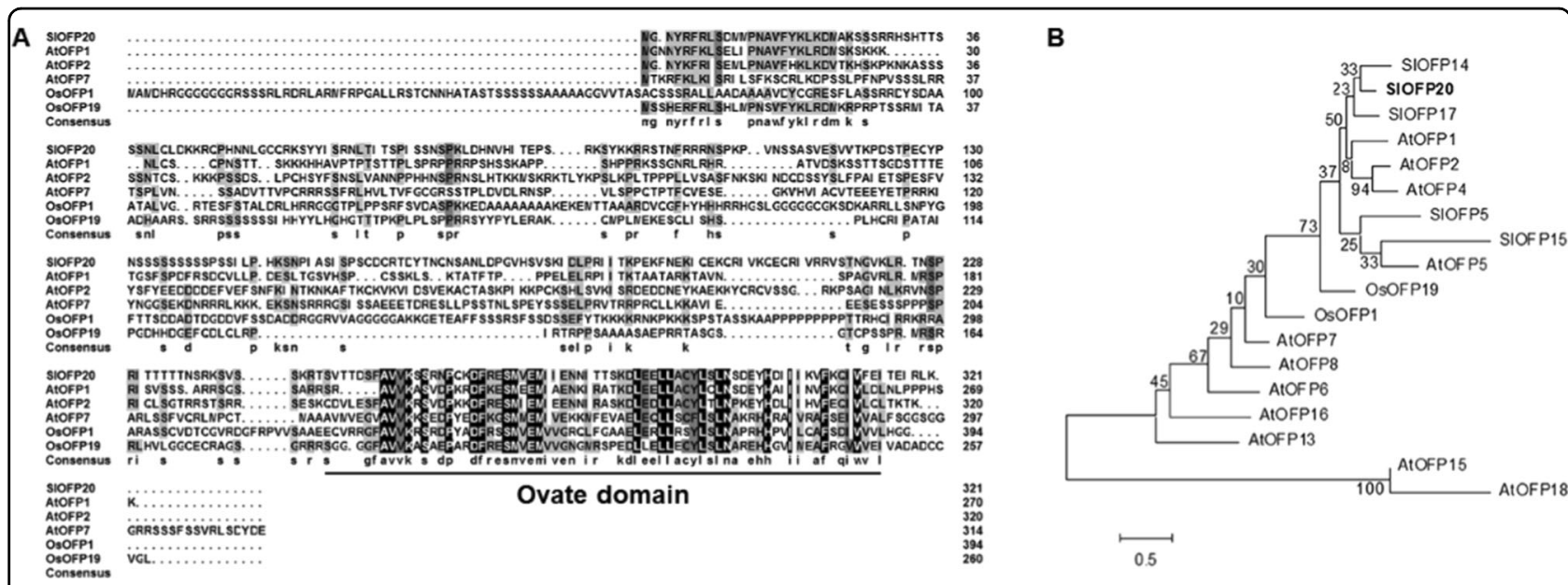

Fig. 1 Amino acid sequence comparison and phylogenetic tree of SIOFP20 and other OVATE family proteins. a Multiple sequence alignment of the SIOFP20, AtOFP1, AtOFP2, AtOFP7, OsOFP1, and OsOFP19 proteins. The black and gray shading show amino acids that are identical or similar, respectively. The conserved OVATE domain in the C-terminus is underlined. $\mathbf{b}$ The phylogenetic tree of SIOFP20 and other OVATE family proteins was constructed to show the relationships between Arabidopsis and rice OVATE family proteins and OVATE family proteins from tomato

from Arabidopsis and rice indicated that the SIOFP20 protein possesses a typical OVATE domain in the Cterminus (Fig. 1a). Phylogenetic analysis was carried out to study the relationship between tomato SIOFP20 and members of the Arabidopsis and rice OVATE family proteins (Fig. 1b), revealing that this tomato protein can be classified into a distinct clade that includes AtOFP1 and OsOFP19, its putative orthologues from Arabidopsis and rice, respectively.

A yeast two-hybrid system was applied to study the transcriptional activity of SlOFP20. A GAL4 DNAbinding domain SIOFP20 fusion protein was expressed in $\mathrm{Y} 2 \mathrm{H}$ yeast cells to evaluate their capacity to initiate transcription from the GAL4 sequence. SIOFP20 could not promote yeast growth in the absence of histidine and adenine (Fig. S1), indicating that SlOFP20 does not exhibit transactivation activity.

\section{Expression patterns of SIOFP20}

To predict the potential function of SlOFP2O underlying tomato growth and development, quantitative reverse transcription-PCR (RT-qPCR) was applied to examine its expression patterns in various tomato organs. As shown in Fig. 2a, the results suggested that SIOFP2O showed the highest transcript accumulation in the roots, followed by the stems and flowers, while relatively low transcript levels were present in the leaves and IMG fruits. SlOFP2O mRNA was not detected in MG, B, B+4, and B+7 fruits. In addition, the expression level of SlOFP2O in the fourwhorl flower organs in WT tomato was analyzed, indicating that SIOFP2O was mainly present in the sepals, stamens, and carpels (Fig. 2b). These results suggested that SlOFP20 showed tissue-specific expression in tomato and may be involved in the development of roots, stems, and flowers.

\section{Overexpression of SIOFP2O alters tomato flower and fruit morphology}

Previous studies on OFP members in Arabidopsis revealed that single or multiple knockout mutants of OFP members do not display morphological defects ${ }^{9,12}$. In contrast, overexpression of some OFP members generates obvious morphological alterations, indicating that these family members have redundant functions. There are 31 OFP family members in tomato, and SIOFP2O is closely related to SIOFP14. In addition, the Atofp1-1 mutant, in which the putative orthologous gene of SIOFP2O in Arabidopsis is mutated, does not show evident morphological changes. Downregulation of SlOFP2O in wild-type tomato does not impact fruit shape ${ }^{25}$. Thus, we inferred that SIOFP20 may exhibit functional redundancy with other OFP members in tomato. For this reason, we overexpressed SIOFP2O in wild-type tomato to investigate its possible functions in tomato growth and development. The full-length SlOFP20 gene fragment was cloned into a plant overexpression vector (pBI121), which was then transferred to WT tomato. Nine independent transgenic lines were obtained. The mRNA accumulation of SlOFP2O was upregulated in the leaves of all transgenic lines. The 35S:SlOFP2O plants exhibited numerous morphological defects related to vegetative and reproductive organs, indicating a prominent role of SlOFP2O in a wide range of tomato growth and developmental phases. One of the most distinct alterations was plant sterility, observed in the strong overexpression transgenic lines. Thus, we selected the mild overexpression transgenic lines OE3, 

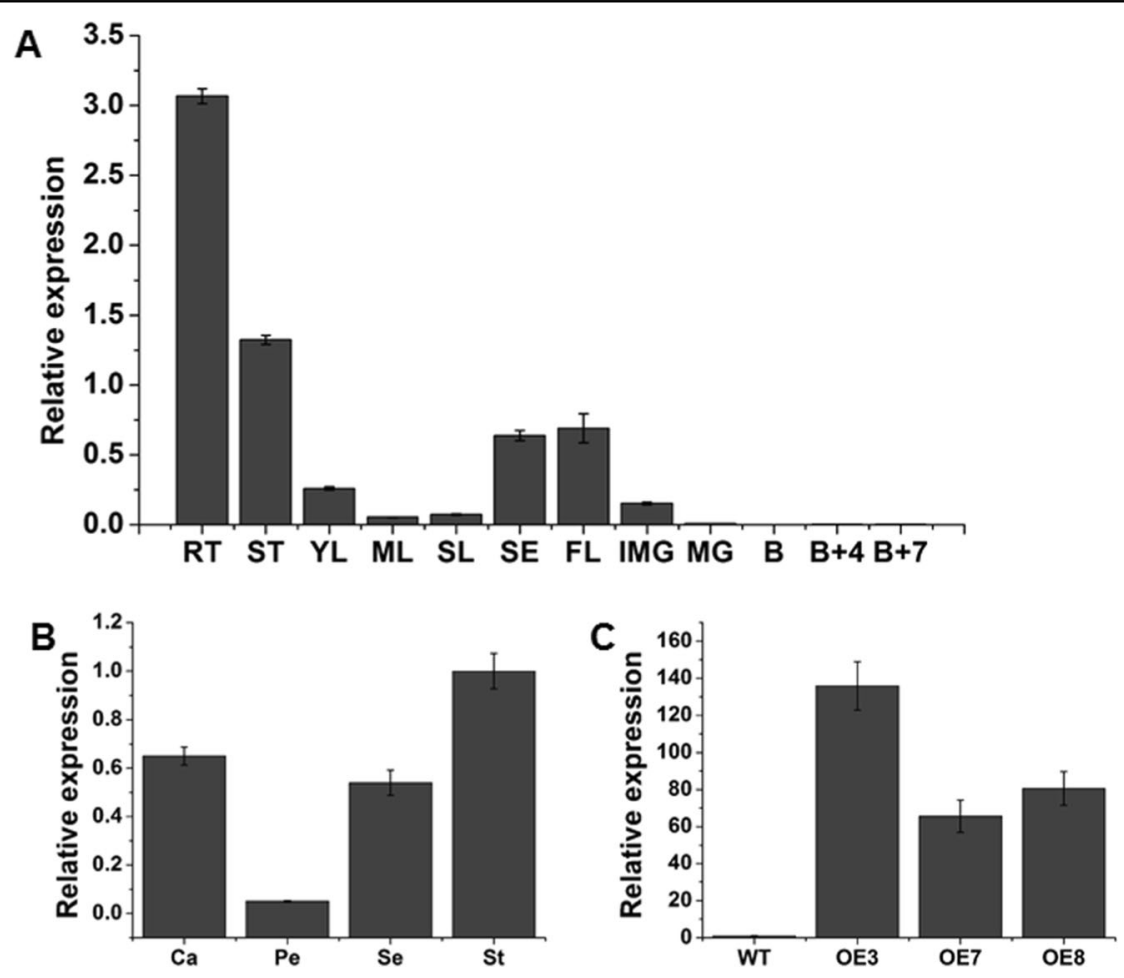

Fig. 2 Expression profile analysis of SIOFP2O in WT tomato plants by quantitative real-time PCR. a Expression levels of SIOFP20 genes in different tissues of WT tomato. Rt, root; St, stem; Yl, young leaf; MI, mature leaf; Sl, senescent leaf; S, sepal; Fl, flower; IMG, immature green fruit; MG, mature fruit; $\mathbf{b}$ breaker fruit; $\mathrm{B}+4,4$ days after the breaker stage; $\mathrm{B}+7,7$ days after breaker stage. $\mathbf{b}$ Relative expression of SIOFP2O in the four-whorl floral organs of WT. Se, sepal; Pe, petal; St, stamen; Ca, carpel. c The expression levels of SIOFP20 in the WT and transgenic lines. Each value represents the mean \pm SE of three replicates

OE7, and OE8, which produced seeds, for further investigation. The representative overexpression efficiency of the T1 generation of OE3, OE7, and OE8 transgenic lines was evaluated by RT-qPCR (Fig. 2c). We also identified strong overexpression plants in the T1 generation transgenic lines, which showed a number of abnormal phenotypes, including plant growth retardation, exserted stigmas, and an altered vegetative and floral architecture. In this study, we focused on these phenotypes associated with reproductive development. Compared to WT, the flowers of OE plants were shorter, but the floral organs, including the sepals, petals, and stamens, were wider (Fig. $3 \mathrm{a}-\mathrm{c})$. Therefore, the length and width of the sepals, petals, and stamens in WT and OE3 plants were measured (Fig. 3g, h). The results showed that the lengths of the sepals, petals, and stamens were significantly reduced in OE3 plants, while the widths were greater than in WT. The shape indexes (length/width) of the sepals, petals and stamens was also calculated, which showed a remarkable reduction in OE3 plants (Fig. 3i). As shown in Fig. 3c, the flowers of $\mathrm{OE}$ line plants showing strong overexpression exhibited exserted stigmas, which prevented pollination and resulted in sterility; thus, we could not observe a phenotype related to tomato fruit. However, the mild overexpression plants in the $\mathrm{T} 1$ generation transgenic lines could produce fruits, and the changes in fruit shape resembled those in the flowers (Fig. 3e, f).

To determine the cytological difference between WT and SlOFP2O-OE flowers, anatomical analysis of the flowers at anthesis was conducted. Compared to WT, the transverse sections of OE3 sepals, petals, and stamens were much thicker due to an increase in the cell layer number and a larger cell size (Fig. 4).

\section{Overexpression of SIOFP2O in tomato affects BR- and GA- related genes}

$\mathrm{BR}$ and GA are two principal phytohormones that function redundantly in promoting plant growth. The most visible phenotype of BR- and GA-deficient plants is dwarfed growth. Here, the phenotypes caused by overexpression of SLOFP2O in tomato strongly resembled those of plants lacking BR and GA. Overexpression of AtOFP1 in Arabidopsis results in reduced cell elongation, which has been partially attributed to the suppression of gibberellin biosynthesis ${ }^{7}$. Moreover, a recent study showed that OsOFP19 negatively regulates the brassinosteroid (BR) response in rice ${ }^{15}$. EBR treatment continuously inhibited the expression of SlOFP2O (Fig. 5a). 


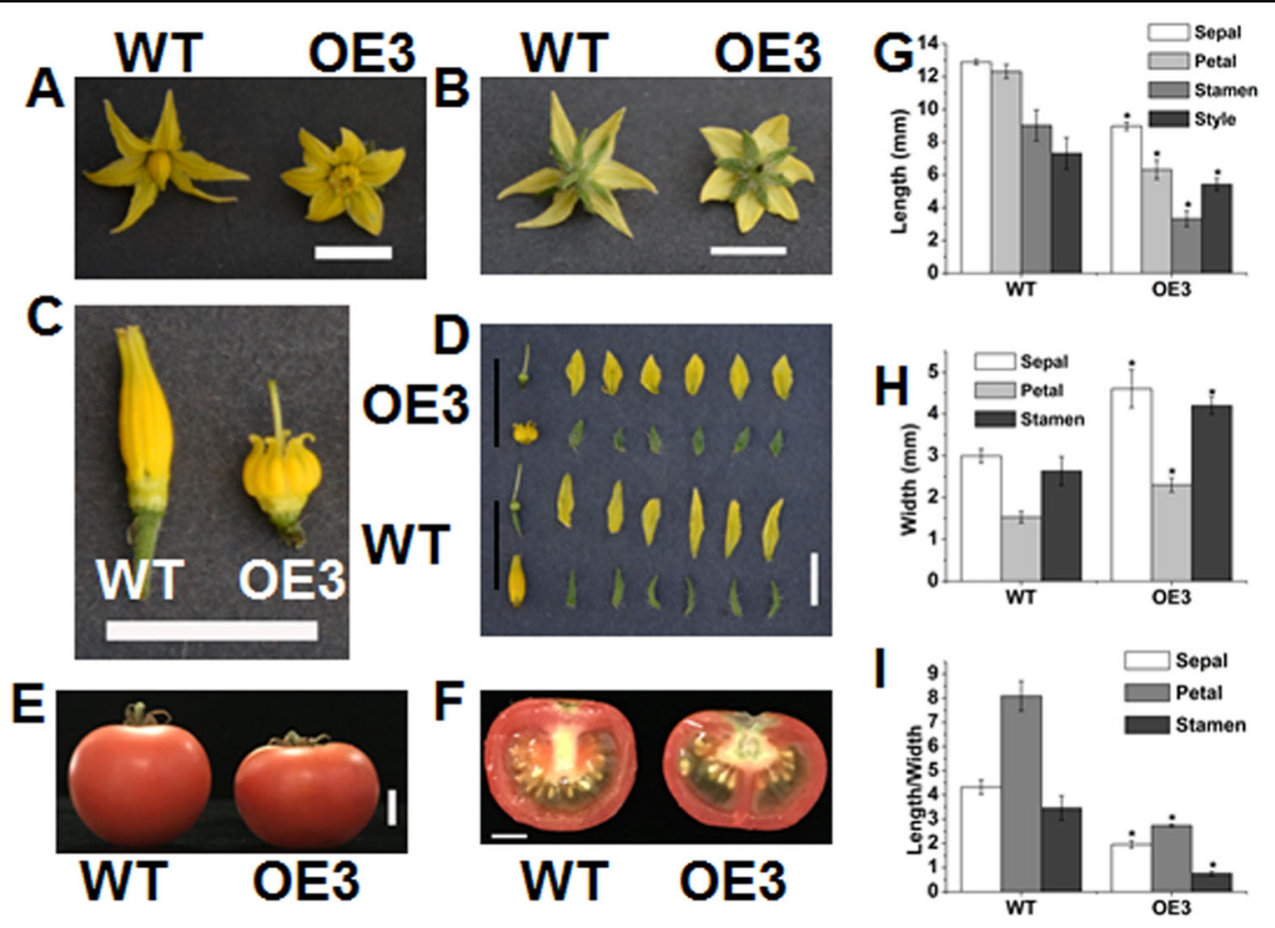

Fig. 3 Morphological changes displayed by SIOFP2O-OE transgenic lines. a, b The overexpression of SIOFP2O resulted in altered flower size. Bar $=1 \mathrm{~cm}$. c Stigma exsertion phenotype of an SIOFP2O overexpression line. Bar $=1 \mathrm{~cm}$. $\mathbf{d}$ The four-whorl floral organs of an SIOFP20 overexpression line (upper row) and WT (lower row). Bar $=1 \mathrm{~cm}$. e, $\mathbf{f}$ The fruit shape of the WT and SIOFP2O overexpression lines. Bar $=1 \mathrm{~cm}$. e, $\mathbf{f}$ Characteristics of fourwhorl floral organs from fully open WT and SIOFP20-OE flowers. $\mathbf{g}, \mathbf{h}$ show the maximum length, maximum width, and shape index of the WT and transgenic lines, respectively. Each value represents the mean \pm SE of three replicates $(n=9) .{ }^{*}$ indicates a significant difference $(P<0.05)$ between the WT and transgenic lines

Therefore, we speculated that overexpression of SlOFP2O inhibited plant growth by controlling the BR and GA pathways. To verify this speculation, the relative expression levels of some BR- and GA-related genes were detected by RT-qPCR. In rice, OsOFP19 increases the transcriptional activity of OSH1 but suppresses $\mathrm{DLT}^{15}$. The homologous genes of OSH1 and DLT in tomato are KONX1 and GRAS41, respectively. Similarly, the transcript level of KNOX1 in SlOFP20-OE transgenic lines was sharply increased (Fig. 5b), whereas the expression of GRAS41 was significantly inhibited compared to that in WT plants (Fig. 5c).

The expression profile of KNOX1 obtained from the Tomato eFP Browser (http://bar.utoronto.ca/efp_tomato/ cgi-bin/efpWeb.cgi) showed that KNOX1 was mainly expressed in roots and flowers (Fig. S2). The GRAS41 transcript also primarily occurred in roots and flowers ${ }^{34}$. SlOFP20, KNOX1, and GRAS41 showed similar expression patterns in tomato, implying that they may function together to regulate plant growth and development. To confirm this possibility, a yeast two-hybrid assay was used to examine their interactions. The results suggested that SlOFP20 could interact with KNOX1 and GRAS41, and KNOX1 and GRAS41 showed a definite interaction (Fig. 5i). In addition, we assessed the mRNA abundance of the BR biosynthesis genes $C P D, D 2$ (an ortholog gene of rice OsD2), and DWARF in SlOFP2O-OE transgenic lines, and the results indicated that the levels of these BR biosynthesis genes were remarkably increased in SIOFP20OE plants (Fig. $5 \mathrm{~d}-\mathrm{f}$ ). Moreover, the BR catabolism gene CYP734A7 was increased in SlOFP20-OE plants (Fig. 5g). The upregulation of $\mathrm{BR}$ biosynthesis genes may be due to feedback regulation, and the BR receptor BRI1 is essential for the homeostasis of endogenous BR contents ${ }^{35}$. Thus, the expression level of BRI1 was measured and was shown to be significantly increased in SIOFP20-OE transgenic lines (Fig. 5h). Similar results were found in OsOFP19-OE transgenic rice plants ${ }^{15}$. Based on these results, we inferred that the mechanism whereby SIOFP20 regulates BR signaling may closely resemble that of its orthologue OsOFP19 from rice.

On the other hand, AtOFP1 reduces the mRNA accumulation of the GA biosynthesis gene AtGA20ox1 via binding to KNAT1, corresponding to KNOX1 in tomato $^{6,7}$. Therefore, we also evaluated the transcript accumulation of GA biosynthesis genes in wild-type and SIOFP20-OE plants. Three genes involved in the early steps of GA biosynthesis, CPS, KS, and $K A O$, were 


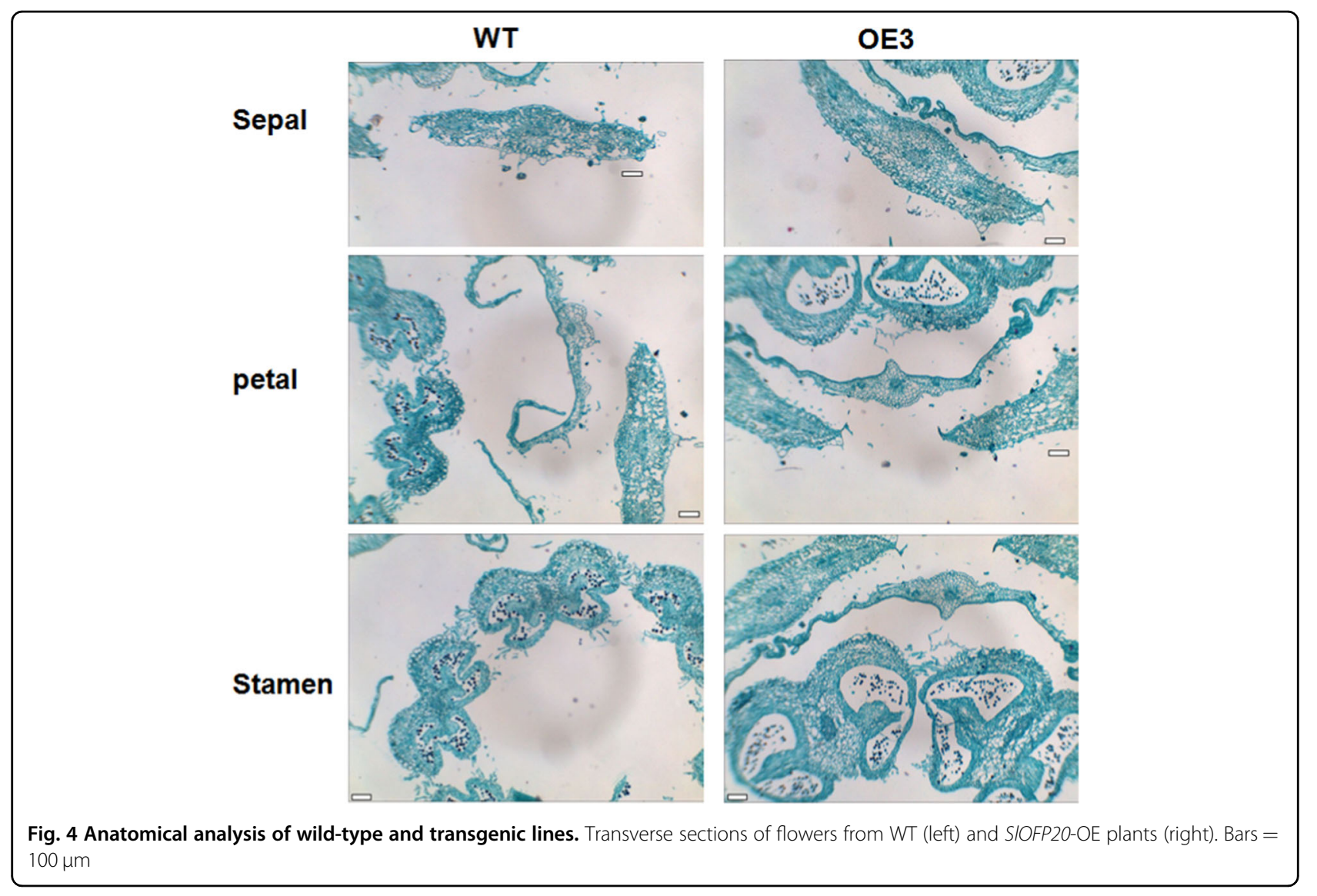

markedly increased in SIOFP20-OE transgenic plants (Fig. $6 \mathrm{a}-\mathrm{c})$. GA20oxs are also major GA biosynthetic enzymes, and GA3oxs catalyze the final step in the generation of bioactive GAs (GA1, GA3, and GA4) ${ }^{36-38}$. The expression levels of GA20ox1, GA3ox1, and GA3ox2 were dramatically increased in SlOFP2O-OE plants compared with wild-type plants (Fig. 6d-f).The expression levels of GA2ox1 and GA2ox2, which encode GA2oxs (the main GA catabolic enzymes) ${ }^{39}$, were also detected, and the data suggested that the levels of both GA2ox1 and GA2ox2 were sharply increased in SIOFP20-OE plants (Fig. 6g, h).

Generally, the length of plant organs is determined by cell number and cell length, which are associated with cell division and cell elongation, respectively. There are hundreds of target genes downstream of the BR and GA pathways, including cell division and cell elongation genes; thus, we attempted to detect the transcript levels of some genes associated with cell division and cell elongation by RT-qPCR. The cell cycle regulatory gene CDKA1 was distinctly downregulated in SIOFP20-OE transgenic lines (Fig. 7a). The transcription accumulation of four cyclin genes was checked. $S l C y c A 3 ; 1, S l C y c B 2$, and $S l C y c D 2 ; 1$ were notably repressed in SlOFP20-OE transgenic lines (Fig. 7b, d, e), but $S l C y c B 1 ; 1$ (Fig. 7c) was not affected. We also detected the mRNA transcript levels of
E2FA and SlCYCT1;3 (Fig. 7f, g), two cell cycle-associated genes that participate in the $\mathrm{G} 1$ to $\mathrm{S}$ transition, but no distinct changes were found between the WT and SIOFP20-OE transgenic lines. The PRE (Paclobutrazol resistance) family of small helix-loop-helix (HLH) proteins positively regulates plant cell elongation ${ }^{40,41}$. There are five putative PREs (PRE1-5) in tomato, and the expression levels of these five genes were examined (Fig. $8 \mathrm{a}-\mathrm{e})$; compared to WT, all of them were notably suppressed in the SIOFP20-OE transgenic lines.

\section{Overexpression of SIOFP2O reduces male fertility}

In this study, we found that the strong SlOFP2O-OE tomato plants could not bear fruit, which may be due to the exserted stigmas of SIOFP2O-OE tomato flowers. We wondered whether the development and function of the pollen in SlOFP20-OE tomato flowers was also affected. The quality of pollen is crucially important in the reproductive stage of most plant species. Hence, a pollen germination experiment was carried out to evaluate the impact of SLOFP2O overexpression on pollen vitality. The results suggested that the pollen germination rate of transgenic plants was distinctly lower than that of WT plants (Fig. 9a, b). Further statistical analysis indicated that the pollen germination rate of SLOFP2O-OE plants 


\section{A}
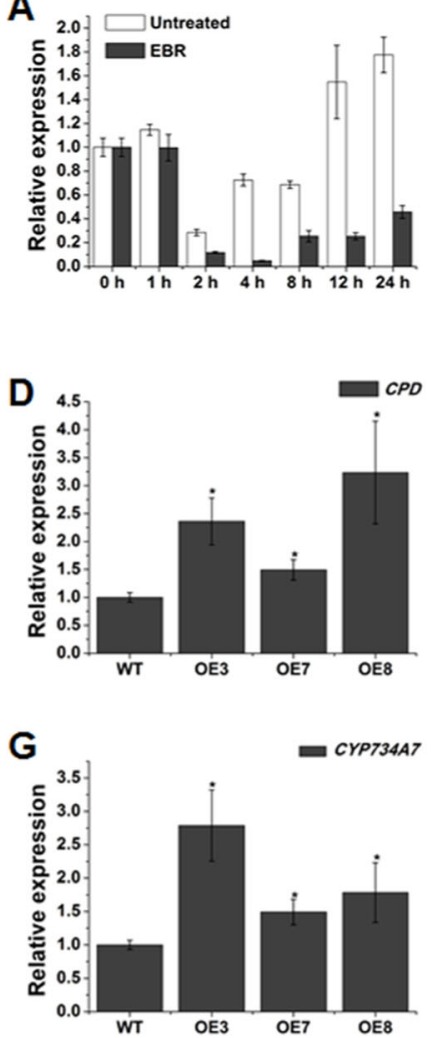

B
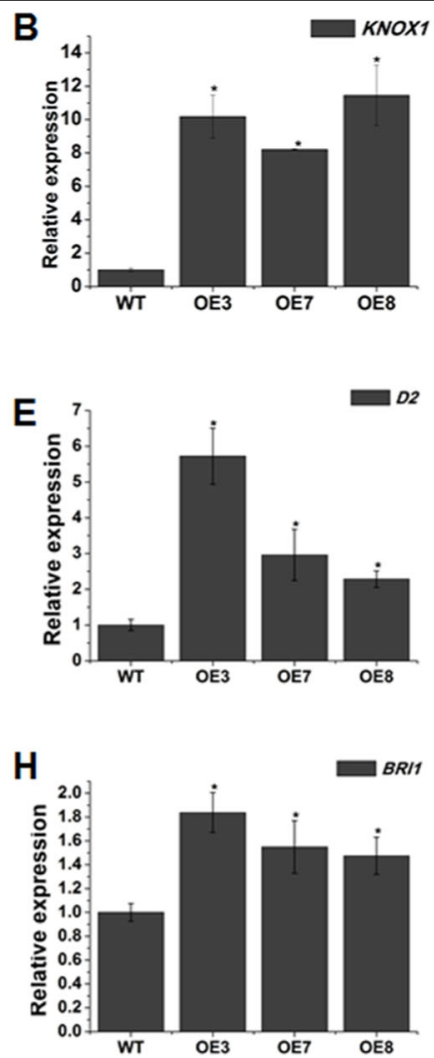

C

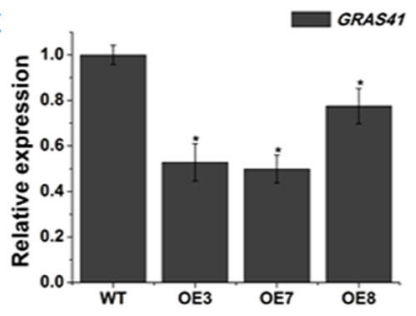

$\mathbf{F}$
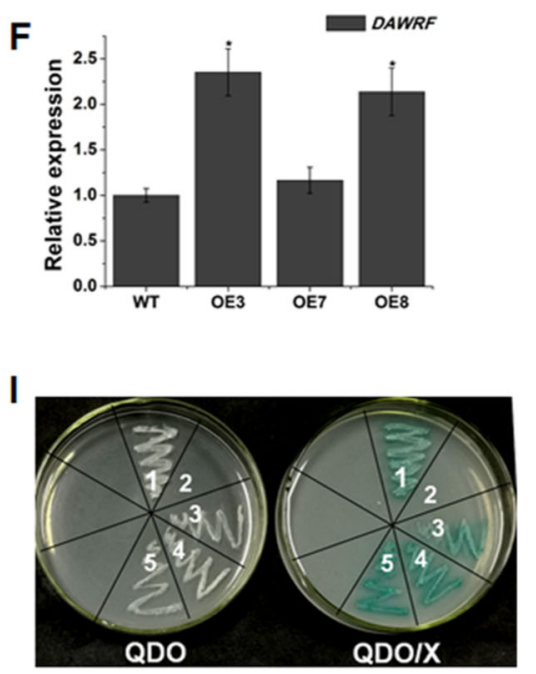

Fig. 5 Overexpression of SIOFP20 affects BR-related genes. a The expression levels of SIOFP20 at different time points under $10 \mu M$ EBR treatment. $\mathbf{b}-\mathbf{h}$ Comparison of BR-related gene expression between WT and overexpression lines. Each value represents the mean \pm SE of three replicates. * indicates a significant difference $(P<0.05)$ between the wild-type and transgenic lines. $\mathbf{i}$ Yeast two-hybrid assay for the SIOFP20 \& SIKNOX1, SIOFP20\& SIGRAS41 and SIKNOX1 \& SIGRAS41 proteins. QDO, SD medium without Trp, Leu, His, and Ade; QDO/X, QDO medium with X-aGal. 1. pGBKT7-53 \& pGADT7-T (positive control); 2. pGBKT7-Lam \& pGADT7-T (negative control); 3. pGBKT7-SIOFP20 \& pGADT7-SIKNOX1; 4. pGBKT7SIOFP20 \& PGADT7-SIGRAS41; 5. pGBKT7-SIKNOX1\& pGADT7-SIGRAS41; Empty bait vector, empty prey vector, and autoactivation assay with no growth of yeast

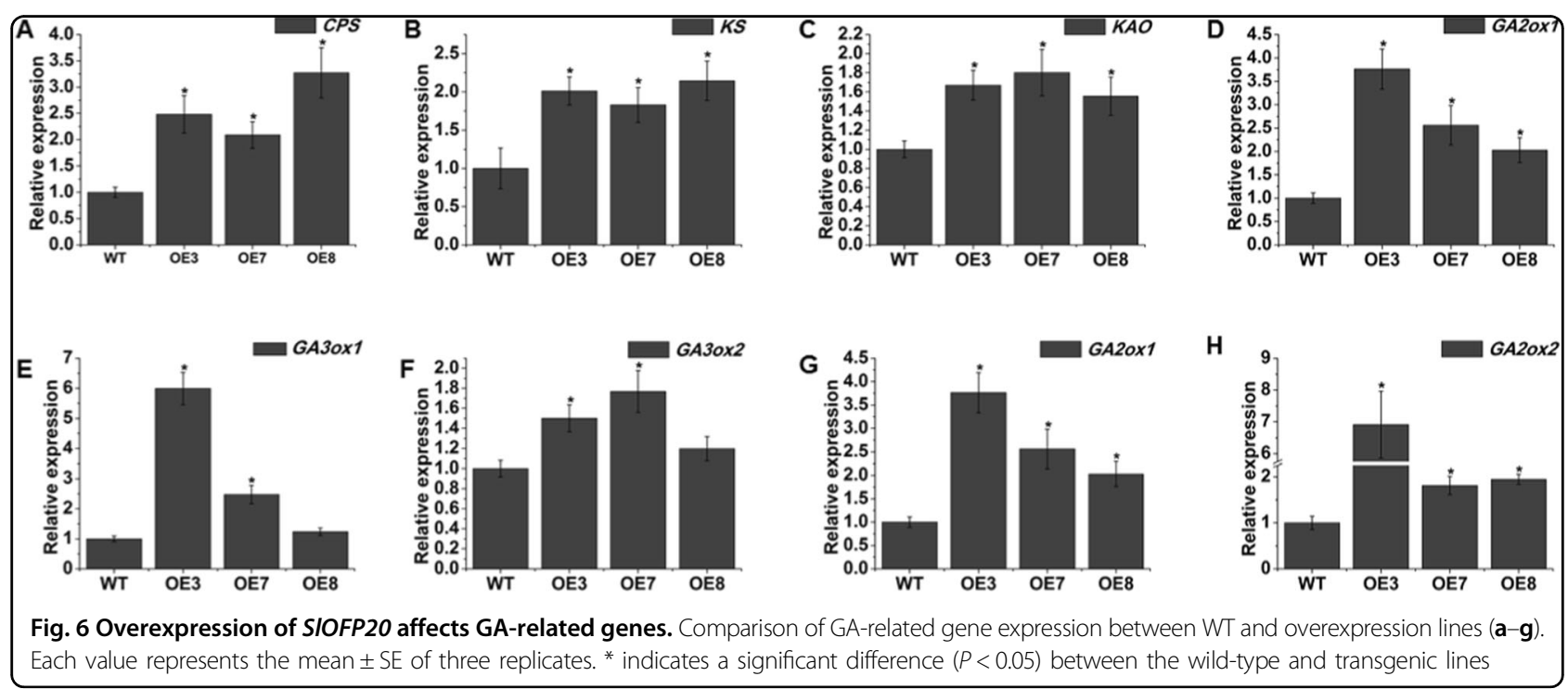



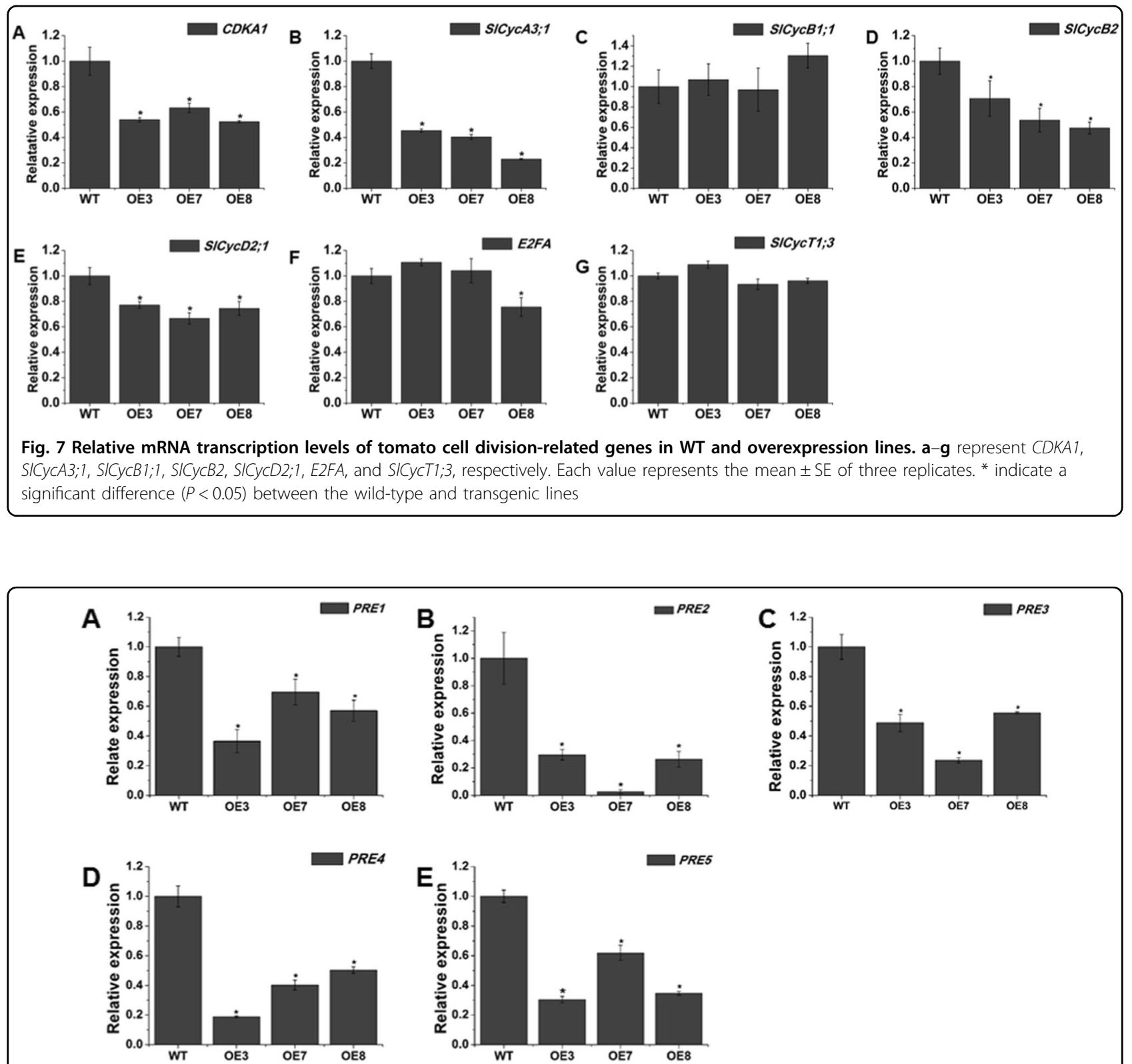

Fig. 8 Expression levels of genes involved in cell elongation in WT and transgenic plants. a-e represent PRE1, PRE2, PRE3, PRE4, and PRE5, respectively. Each value represents the mean \pm SE of three replicates. ${ }^{*}$ indicates a significant difference $(P<0.05)$ between the wild-type and transgenic lines

was 2.36-fold lower than that of WT plants (Fig. 9e). Moreover, TTC staining for pollen viability suggested that there were fewer pollen viable grains in SlOFP20-OE flowers than in WT flower. These results suggested that overexpression of SlOFP2O may impair male fertility. When the flowers of SIOFP20-OE plants were manually crossed with WT pollen, normal seeds could develop, hinting that the female fertility of SlOFP20-OE transgenic plants may not be affected (Fig. 9f).

A scanning electron microscope was employed to observe the stamen morphologies of WT and transgenic plants. The stamen epidermal cells of the transgenic plants were shorter and more intense than those of WT, and the cell arrangement in OE3 had a scale-like appearance (Fig. 10a, b). The shape of the pollen grains in WT and the OE3 transgenic plants did not show obvious changes (Fig. 10c, d). The grains of both plant lines presented a normal globular shape. In addition, we detected three floral organ identity genes, TAG1, TAGL2, and TM5. TAG1 is a C-class gene that has been suggested to take part in the specification of stamen and carpel identities ${ }^{42}$. TM5 $5^{43}$ and TAGL2 (syn. $T M 29)^{44}$ are E-class genes. The expression levels of TAG1 

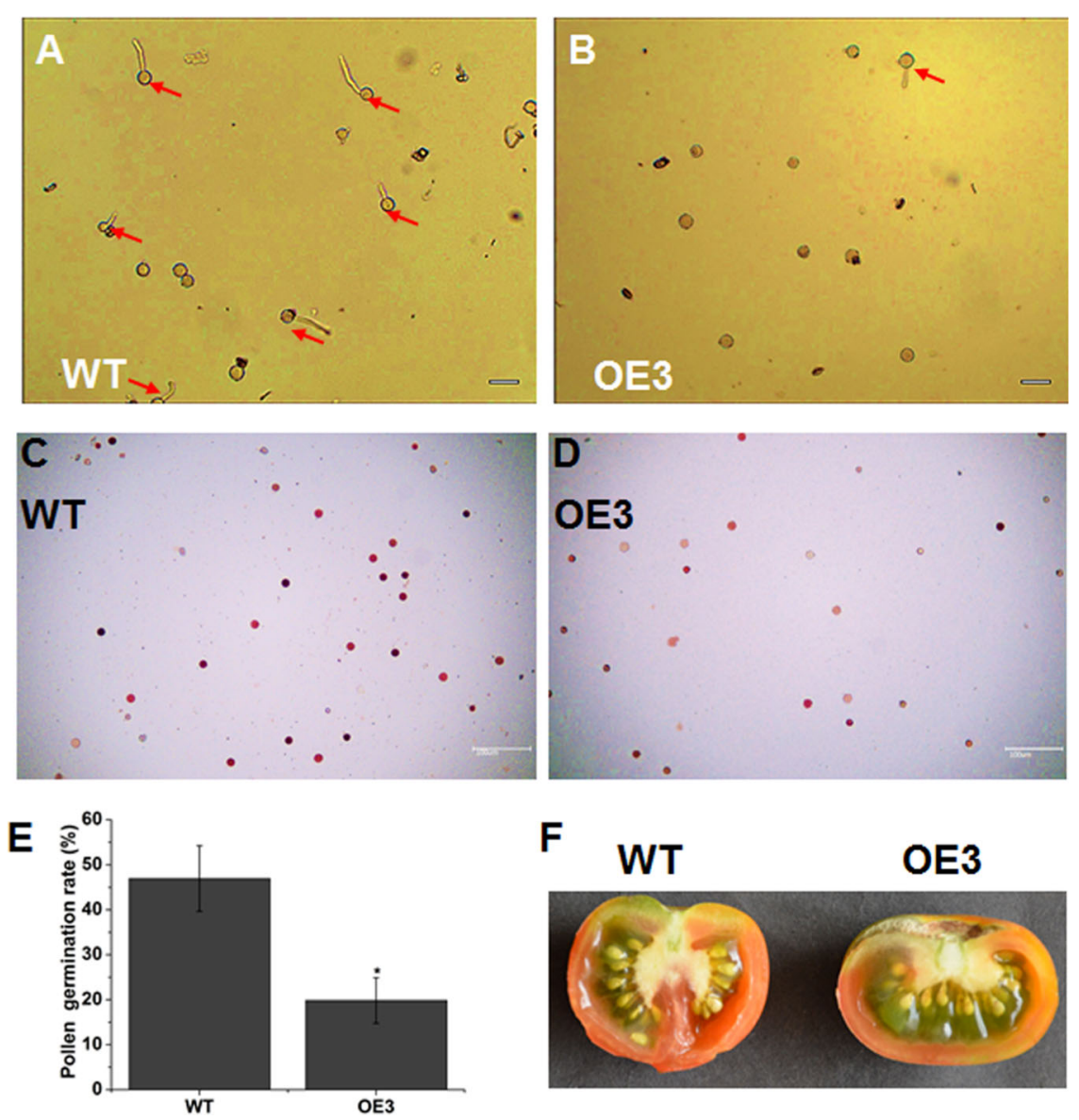

Fig. 9 Overexpression of SIOFP20 reduces male fertility. Pollen germination of WT (a) and SIOFP2O-OE lines (b). The red arrow indicates germinated pollen. Bars $=50 \mu \mathrm{m}$. Comparison of the pollen germination of WT (c) and SIOFP2O-OE (d) with TTC staining. Bars $=100 \mu \mathrm{m}$. e Comparison of pollen germination rates between WT and SIOFP2O-OE lines. $\mathbf{f}$ The fruit of the transgenic line generates normal seeds in a manual crossing assay

and TAGL2 were not obviously altered in SIOFP20-OE transgenic lines (Fig. 10e, g), while that of TM5 was evidently increased in SlOFP20-OE transgenic plants (Fig. 10f).

Moreover, the expression levels of pollen developmentspecific genes were evaluated in WT and SLOFP20-OE tomato plants. SlCRK1, a cysteine-rich receptor-like kinase, plays a critical role in pathogen protection and programmed cell death ${ }^{45}$. Pectin methylesterase inhibitor (SIPMEI) acts as a key regulator of pectin methylesterase $(\mathrm{PME})^{46}$. LePRK3, a pollen-specific receptor kinase gene, may take part in perceiving extracellular cues during pollen tube growth ${ }^{47}$. SIPRALF, an exogenous rapid alkalinization factor, negatively adjusts the elongation of the pollen tube ${ }^{48}$, and LAT52 may participate in germination or early tube growth ${ }^{49}$. The results showed that all five of these genes were remarkably repressed in SIOFP2OOE lines, indicating that the overexpression of SIOFP2O suppressed the mRNA expression of these genes, resulting in reduced pollen fertility (Fig. 11a-e).
Mounting evidence suggests that numerous cis-elements play a vital role in specifying the tissue expression patterns of plant genes ${ }^{50,51}$. To understand what drives the expression of SLOFP2O in the pollen, a 2000-bp region upstream of the SIOFP2O start codon was submitted to a public database (http://www.dna.affrc.go.jp/PLACE) to analyze cis-acting elements to determine whether pollen developmentassociated elements were present in the promoter. There were ten pollen-specific activation-related elements POLLEN1LELAT5 $2^{52}$ and ten late pollen gene g10-related elements $^{53}$ identified in the SIOFP2O promoter (Supplementary Table S2). This result further demonstrated that SlOFP2O may participate in pollen development.

\section{Mild overexpression of SIOFP2O in tomato may promote seed germination}

In this study, the plants with mild overexpression of SlOFP20 could generate fruits with seeds. However, compared to WT, the seed number of OE3 transgenic plants 

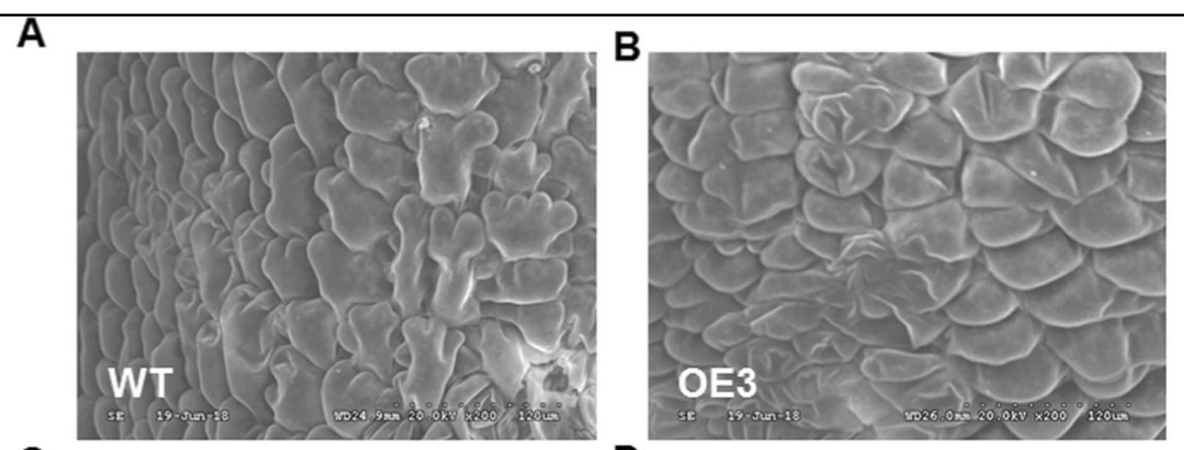

C
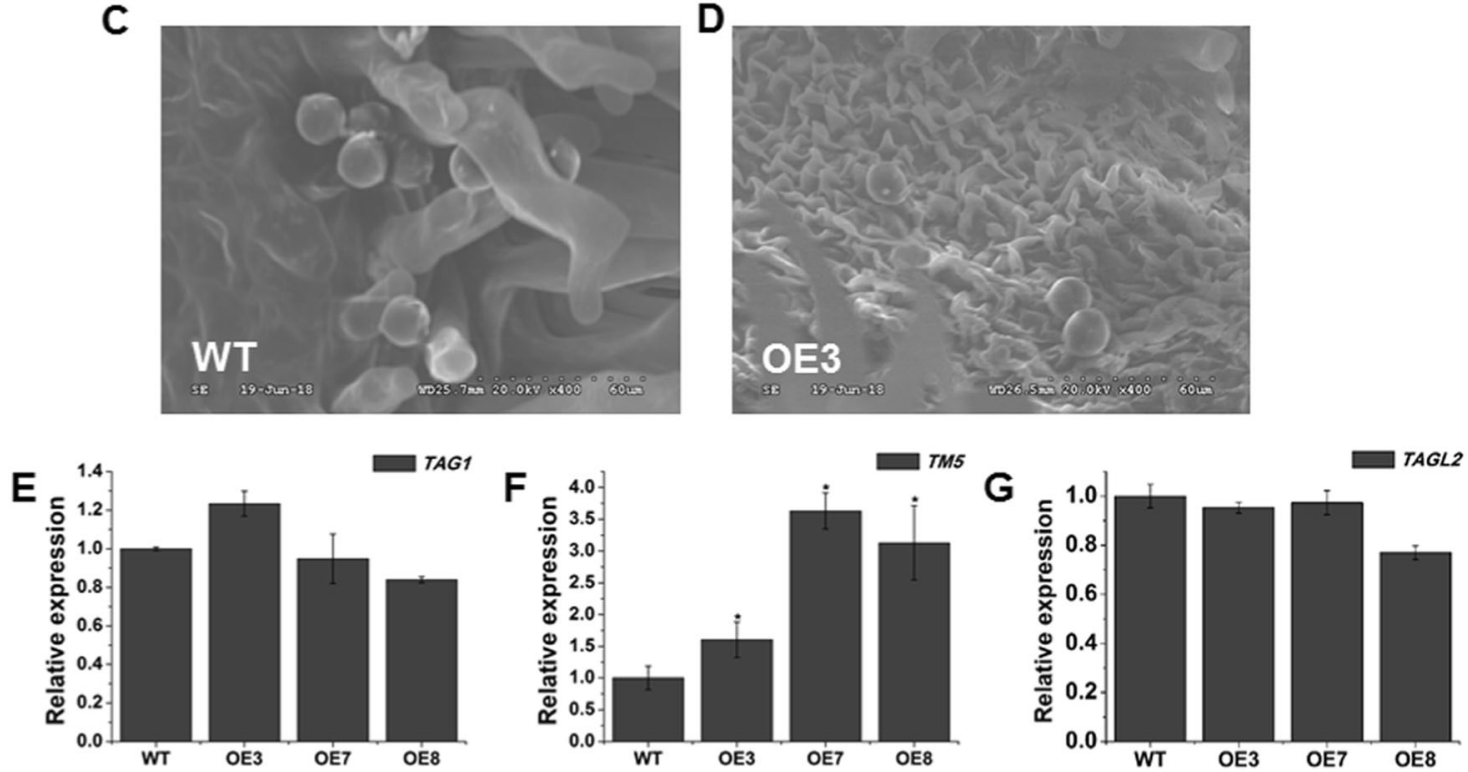

Fig. 10 Overexpression of SIOFP2O affects stamen morphology. Overexpression of SIOFP2O affects stamen morphology. Electron microscopy observations of epidermal cells of the stamen and pollen in WT $(\mathbf{a}, \mathbf{c})$ and SIOFP2O-OE transgenic lines $(\mathbf{b}, \mathbf{d})$. $\mathbf{a}, \mathbf{b}$ Morphology of epidermal cells of the stamen. $\mathbf{c}, \mathbf{d}$ Morphology of pollen grains. The expression levels of floral organ identity genes in WT and SIOFP20-OE lines. TAG1 (C-class gene) (a), TM5 (b) and TAGL2 (E-class genes) (c) in WT and transgenic lines. Each value represents the mean \pm SE of three replicates. ${ }^{*}$ indicates a significant difference $(P<0.05)$ between WT and transgenic lines

was reduced by $\sim 49 \%$, which may be partially due to the reduced male fertility of SIOFP20-OE transgenic plants (Fig. 12a). As the seed number was decreased in the transgenic plants, we sought to determine the germination energy of transgenic tomato seeds; thus, a seed germination experiment was performed. The results indicated that SIOFP20$\mathrm{OE}$ transgenic seeds exhibited notably higher germination rates than those of WT (Fig. 12b, c), implying that mild overexpression of SIOFP2O in tomato may speed up transgenic tomato seed germination.

\section{Discussion}

The OVATE gene is initially found in tomato and shown to act as a plant-growth suppressor ${ }^{5}$. Extensive studies have since focused on the functional analysis of this family in Arabidopsis and rice. As this research has progressed, OVATE family proteins have come to be considered vital regulators involved in organ shape and size determination $^{5,9}$, secondary cell wall formation ${ }^{10}$, embryo sac development ${ }^{19}$, fruit ripening ${ }^{16,17}$, vasculature development $^{12}$, vegetative to reproductive phase transition $^{11}$, male transmission and pollen function ${ }^{6}$, and phytochrome signaling ${ }^{7,13-15}$. However, since the functional description of OVATE, little attention has been paid to the other OVATE family proteins in tomato. SIOFP20, a member of the OVATE family proteins, was isolated and functionally studied by using overexpression technology. Overexpression of SLOFP2O in wild-type tomato distinctly impacted the development of the vegetative and reproductive phases. In this paper, we were particularly concerned with the influence of SlOFP2O on the regulation of reproductive development. The strong overexpression of SlOFP2O in tomato resulted in exserted stigmas, an altered floral architecture and an absence of fruit, indicating that the overexpression of SlOFP2O affected the development of reproductive processes.

Overexpression of OVATE produces pleiotropic phenotypes in tomato plants, including exserted stigmas, 

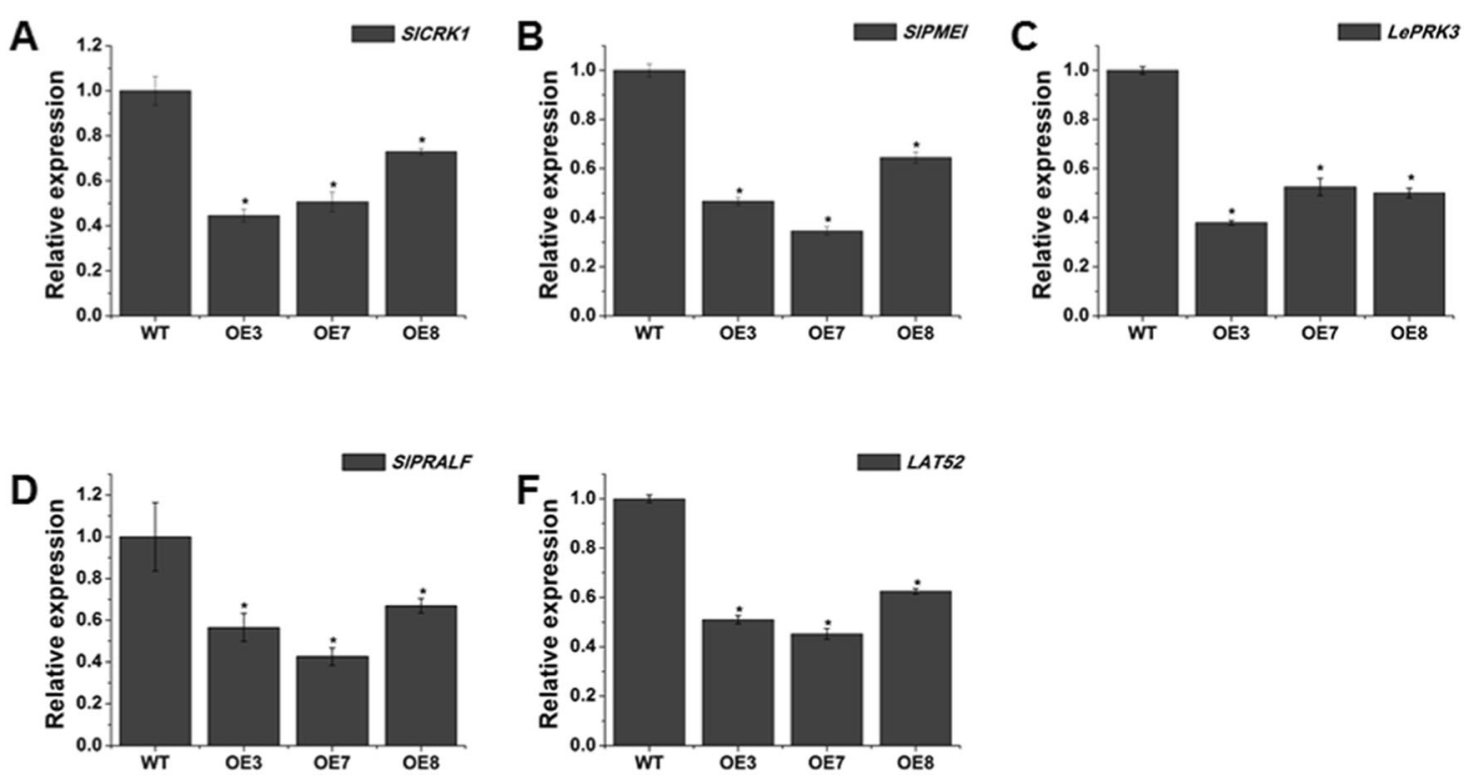

Fig. 11 Expression analysis of tomato pollen-specific genes in the pollen of WT and transgenic plants. (a-e) represent the expression of the pollen-specific genes SICRK1, SIPMEI, LePRK3, SIPRALF and LAT52 in the pollen of WT and transgenic lines. Each value represents the mean \pm SE of three replicates. ${ }^{*}$ indicates a significant difference $(P<0.05)$ between $W T$ and transgenic lines
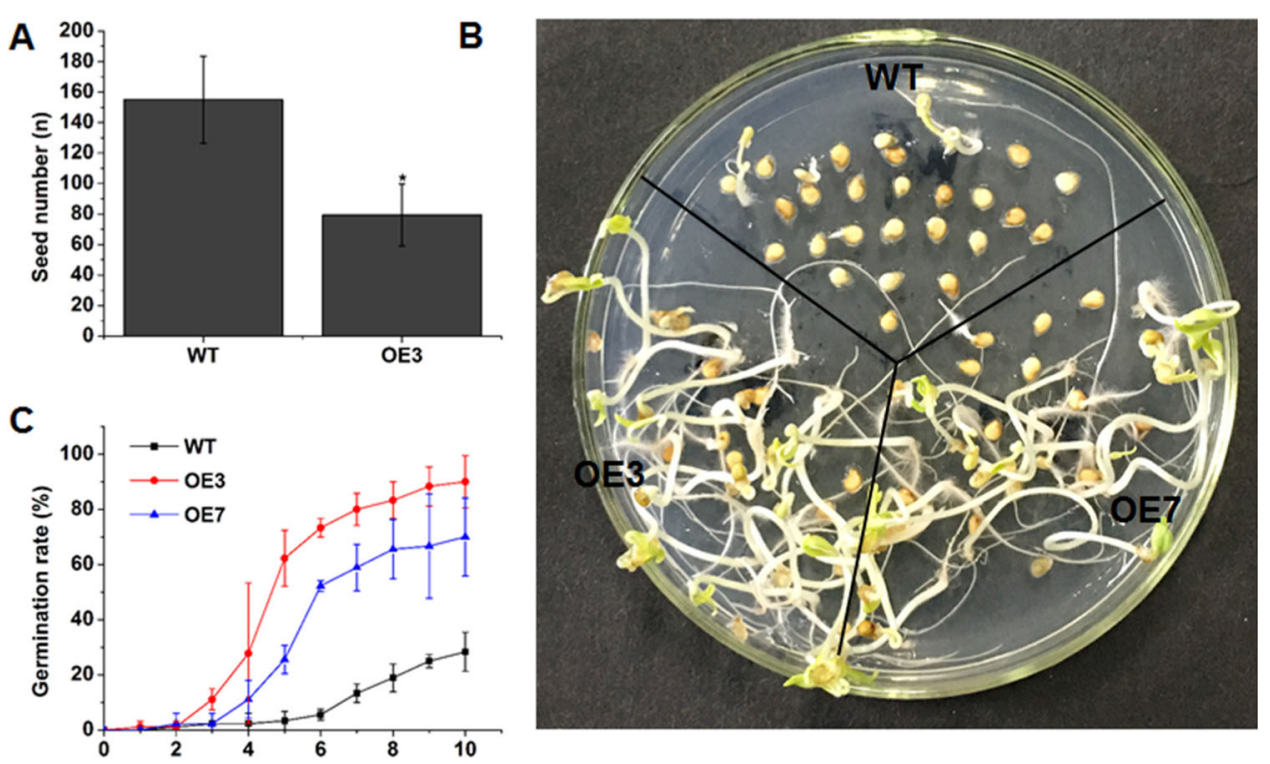

Fig. 12 Mild overexpression of SIOFP2O in tomato may promote seed germination. a The seed number per fruit of WT and mild SIOFP20-OE transgenic plants. Values are presented as the means \pm SEs of measurements from 20 independent fruit. b Germination phenotype of WT and SIOFP2O-OE transgenic seeds. c Germination rates of seeds on MS medium. Seed germination was scored every day. The data are the means \pm SEs from three replicates with 30 seeds per replicate

smaller floral organs, and round fruit ${ }^{5}$. In our study, the floral architecture of the strong SlOFP2O overexpression lines resembled that of OVATE overexpression lines. Similar phenotypes related to flowers have been found in Arabidopsis $^{7}$ and rice $^{15}$, indicating that OFPs exhibit overlapping functions in controlling plant growth and development. Statistical data suggested that the sepals, petals, and stamens of SIOFP20-OE plants were much shorter and wider than those of WT plants. The exserted stigma phenotype of strong SIOFP2O-OE plants may be 
ascribed to uneven repression of the growth of different floral organs, similar to what is observed upon the overexpression of ovate in tomato.

BRs are a group of polyhydroxylated steroid hormones that play central roles in controlling plant growth and development and conveying various environmental inputs $^{54,55}$. The primary BR signaling pathway has been well established in Arabidopsis. Briefly, BRs directly bind to BRI1 (BRASSINOSTEROID-INSENSITIVE1), which belongs to the plasma-membrane-localized and leucinerich repeat (LRR) receptor kinases ${ }^{56-58}$, thereby activating it and triggering a signal transduction cascade. Finally, the accumulation of unphosphorylated transcription factors BZR1/ BES1 in the nucleus modulates BR-responsive genes by binding target $\mathrm{DNA}^{59,60}$. OsOFP $1^{14}$, OsOFP $8^{13}$, and OsOFP $19^{15}$ have been proposed to take part in the regulation of BR signaling. Considering the similar phenotypes observed in SlOFP2O-OE tomato plants and OsOFP19-OE rice plants, we wondered whether the regulatory mechanisms of SIOFP20 and OsOFP19 affecting plant growth and development were conserved in tomato and rice. Ectopic expression of the rice homeobox gene OSH1 in tobacco generates thicker and shorter leaves ${ }^{61}$, and loss-of-function of DWARF AND LOW-TILLERING (DLT) results in a semidwarf phenotype with wider and shorter leaves ${ }^{62}$. OsOFP19 directly interacts with OSH1 to increase the transcriptional activity of OSH1, resulting in a transition of the cell division pattern and antagonizing DLT in BR signaling, which positively regulates BR signaling ${ }^{15}$. Thus, we attempted to examine the transcript levels of the homologous genes of OSH1 and DLT in tomato, KNOX1 and GRAS41. The results suggested that the mRNA accumulation of KNOX1 in SlOFP20-OE transgenic lines was sharply increased. In contrast, the mRNA accumulation of GRAS41 was notably decreased. We also tried to examine the interactions between SlOFP20, KNOX1, and GRAS41 by using a yeast two-hybrid assay, and positive results were obtained. KONX1 and GRAS41 exhibited a clear interaction, indicating that SIOFP20, KNOX1, and GRAS41 can form a complex. To determine the influence of the overexpression of SIOFP2O on BR metabolism, we assessed the expression of $C P D, D 2$, and $D W A R F$, which are key genes for BR biosynthesis. The expression of $C P D, D 2$, and $D W A R F$ was significantly promoted in SIOFP20-OE transgenic plants. In addition, we evaluated the transcript abundance of CYP734A7, encoding a key BR catabolic enzyme. The transcript level of CYP734A7 was also increased in SIOFP20-OE transgenic plants. These results are consistent with observations made in OsOFP19-OE transgenic plants. In addition, we noted that the upregulation of BR biosynthesis genes may be due to feedback regulation by $B R$ signaling, which was not discussed in relation to OsOFP19-OE transgenic plants. OsOFP19-OE plants show greatly reduced sensitivity to 24- epibrassinolide treatment, indicating that OsOFP19 plays a negative role in the $\mathrm{BR}$ response ${ }^{15}$. In rice, OSH1 promotes the mRNA accumulation of BR degradation-related genes, and inducible overexpression of OSH1 results in insensitivity to $\mathrm{BR}^{63}$. Previous studies have suggested that BR-insensitive mutants exhibit increased transcript abundance of BR biosynthesis genes and a higher BR content ${ }^{35,64}$. BRI1 is essential for the homeostasis of endogenous BR contents ${ }^{35}$. Therefore, we measured the transcript level of $B R I 1$, which was notably increased in SIOFP20-OE transgenic plants. Similar characteristics are found in the rice $d l t$ mutant, which also shows less sensitivity to BRs. The BR biosynthesis genes D2, D11, OsCPD, and OsBR6ox are all upregulated, as is the BR signaling gene $B R I 1^{62}$. In our study, the GRAS41 gene homolog of $D L T$ exhibited reduced levels in transgenic plants. Whether KNOX1 and GRAS41 participate in BR signaling has not yet been reported in tomato. Based on the above results, we speculate that SIOFP20 may negatively regulate the BR response in tomato, similar to OsOFP19 in rice. In addition, the altered floral architecture of SLOFP2O-OE plants may mainly be due to the reduced $B R$ response.

The phenotypes of BR-deficient or BR-insensitive mutants are similar to those of GA-deficient or GAinsensitive plants. Many studies have focused on the question of whether BRs may regulate growth by impacting GA biosynthesis. BR treatment and overexpression of DWF4 increase the expression of three GA biosynthesis genes, GA20ox1, GA20ox2 and GA20ox5, in Arabidopsis ${ }^{65}$. In addition, BRs have been found to modulate GA biosynthesis in rice ${ }^{66}$. Hence, we assumed that GA metabolism was altered in the SIOFP20-OE transgenic lines. To investigate this hypothesis, the expression levels of GA biosynthesis genes, including CPS, KS, KAO, GA2Oox1, and GA3ox 1 and GA3ox2, were measured. All of these genes showed increases in SlOFP20-OE transgenic plants. Moreover, we detected the mRNA abundance of the GA inactivation genes GA2ox1 and GA2ox2. The expression of GA2ox1 and GA2ox2 was increased. Interestingly, the mRNA accumulation of all of the examined BR and GA metabolism genes was found to be increased in our study. Previous work has shown that high levels of BRs induce GA inactivation by increasing the expression of GA2ox-3 to counter the increase in GA biosynthesis due to increased GA3ox-2 expression, finally resulting in growth inhibition ${ }^{66}$. As stated above, BR-insensitive mutants exhibit a higher content of BR, and SlOFP20 may negatively control BR signaling. Thus, we speculate that overexpression of SLOFP2O in tomato reduces $\mathrm{BR}$ signaling, resulting in the accumulation of $B R$, which then induces the mRNA accumulation of the GA inactivation genes GA2ox1 and GA2ox2 to counteract the increase in GA biosynthesis due to increased expression of CPD, KS, KAO, GA20ox1, GA3ox1, and GA3ox2, ultimately leading to growth inhibition. 
There are hundreds of target genes downstream of the BR and GA pathways, including cell division and cell elongation; thus, the expression levels of cell division genes (CDKA1, SlCycA3;1, SlCycB1;1, SlCycB2, $S l C y c D 2 ; 1, E 2 F A$, and $S l C Y C T 1 ; 3)$ and cell elongation genes $(P R E 1-5)$ were investigated. Four cell division genes, CDKA1, SlCycA3;1, SlCycB2, and SlCycD2;1, were significantly suppressed in the SIOFP20-OE transgenic plants. Overexpression of SIPRE2 in tomato promotes the elongation of plant stem internodes ${ }^{67}$. In our study, the downregulation of SIPRES illustrated that cell elongation may be impaired in SlOFP20-OE transgenic plants. The SEM observations of the stamen surface also supported this result. Therefore, overexpression of SlOFP20 repressed cell division and cell elongation.

The key yield components of most crop species are fruit and seeds. Therefore, extensive studies have focused on fruit and seed development for decades ${ }^{68}$. In our study, transgenic tomato plants with strong overexpression of SlOFP2O bore no fruit. BR-deficient and BR-perceptional mutants, including $c p d, d w f 4$, and bri1, are male sterile or show significantly reduced male fertility due to shortening of the stamen and defects in pollen development, and these developmental defects correlate with the inhibition of several critical genes that participate in the development of anthers and pollen, indicating that BRs are critical for plant reproductive development ${ }^{69-71}$. As mentioned above, overexpression of SlOFP2O reduced the BR response. Here, we also observed reduced length of the stamen, resulting in an exserted stigma phenomenon, which blocked the normal pollination process. On the other hand, a pollen germination experiment was carried out to examine the germination ability between WT plants and SlOFP20-OE transgenic tomato plants. The results showed that the pollen germination rate of the transgenic plants was significantly decreased, and this result was further supported by TTC staining, indicating that overexpression of SIOFP2O inhibits the normal development of pollen grains, but their form was not significantly altered when observed by SEM. In addition, a manual crossing assay was performed to verify that the maternal fertility of SIOFP20-OE transgenic lines was not affected. Furthermore, RT-qPCR analysis was conducted to check the transcript accumulation of tomato pollenassociated genes, including SlCRK1, SlPMEI, LePRK3, SIPRALF, and LAT52. All of these genes showed a trend of downregulation in the transgenic lines, suggesting that SlOFP2O may control the mRNA accumulation of these pollen-specific genes to impact pollen development. In addition, previous studies revealed that many pollenspecific cis-acting elements, including the pollen-specific activation-related elements POLLEN1LELAT52 and the late pollen gene g10-related elements, were enriched in the promoter regions of two pollen-specific genes,
$S l C R K 1^{45}$ and SLPMEI ${ }^{46}$. Promoter-GUS chimeric expression experiments have been used to confirm that the promoters of $S l C R K 1^{45}$ and $S l P M E I^{46}$ exhibit strong pollen-specific activity in the transgenic Arabidopsis and tomato plants. In our study, 10 pollen-specific activationrelated elements POLLEN1LELAT52 and 10 late pollen gene g10-related elements were found in the SlOFP20 promoter, which further suggests that SlOFP2O may play an important role in pollen development. Similarly, AtOFP1 has been demonstrated to be essential for pollen function ${ }^{6}$.

Moreover, tomato plants with mild overexpression of SlOFP2O can bear fruit. However, the seed number per fruit in the transgenic plants is sharply reduced compared with that in WT plants, which is in accord with the impairment of the pollen germination rate in transgenic plants. In addition, a seed germination experiment was performed to assess the quality of SlOFP20-OE transgenic seeds, and the results clearly showed that the germination rate of transgenic plants was higher than that of WT. AtOFP5 has been demonstrated to interact with BLH1 and KNAT3, which guarantees normal embryo sac development in Arabidopsis $^{19}$. Therefore, we assumed that SlOFP20 may participate in the development of the embryo sac, and more elaborate experiments should be performed to verify this possibility in the future.

In conclusion, the major objectives of this study were to investigate the influence of the overexpression of SlOFP2O on the reproductive development of tomato. Overexpression of SIOFP2O in tomato not only altered the morphology of the flowers and fruit but also reduced male fertility. In addition, mild overexpression of SIOFP20 may accelerate the germination rate of transgenic lines. Analyses of morphological, physiological, and molecular features have been performed to preliminarily elucidate the causes of SlOFP20-OE plants defects. A working model is proposed to explain the functions of SIOFP20 in plant growth and development (Fig. S3). Briefly, strong overexpression of SlOFP2O may directly inhibit the BR response via promoting the expression of $K N O X 1$ and suppressing the expression of GRAS41, leading to changes in a large number of genes, such as genes affecting cell division, cell elongation and pollen development. On the other hand, the reduced BR response in SIOFP20-OE plants leads to the accumulation of $\mathrm{BR}$, which then induces the expression of GA inactivation genes to counteract the increase in GA biosynthesis. These results highlight that SIOFP20 functions as an important transcription factor to modulate reproductive development in tomato plants. Thus, it is meaningful to identify the biological function of SIOFP20 or other OVATE family proteins, which will not only extend knowledge of the biological functions of OVATE family proteins but also 
provide new insight for exploring the importance of OFPs in controlling plant vegetative growth and reproductive development. Considering that OFPs have been suggested to share overlapping functions with other OFP gene family members, a gain-of-function approach was used to study the functions of SlOFP2O in this study. In future studies, CRISPR/Cas9 genome editing may be a better way to generate Slofp 20 mutants for gene functional studies. To identify the protein partners and targeted genes of SIOFP20, it can be beneficial to elucidate the molecular mechanisms by which SIOFP20 modulates plant growth and development.

\section{Acknowledgements}

This work was supported by the National Natural Science Foundation of China (no. 31572129) and the Technology System of National Bulk Vegetable Industry-Eggplant Breeding Position (CARS-25-A-06).

\section{Conflict of interest}

The authors declare that they have no conflict of interest.

Supplementary Information accompanies this paper at (https://doi.org/ 10.1038/s41438-019-0207-6).

Received: 25 July 2018 Revised: 4 September 2019 Accepted: 12 September 2019

Published online: 15 November 2019

\section{References}

1. Gimenez, E. et al. TOMATO AGAMOUS1 and ARLEQUIN/TOMATO AGAMOUSLIKE1 MADS-box genes have redundant and divergent functions required for tomato reproductive development. Plant Mol. Biol. 91, 513-531 (2016).

2. Ferreira e Silva, G. F. et al. microRNA156-targeted SPL/SBP box transcription factors regulate tomato ovary and fruit development. Plant J. 78, 604-618 (2014).

3. Huang, B. et al. Overexpression of the class D MADS-box gene SI-AGL11 impacts fleshy tissue differentiation and structure in tomato fruits. J. Exp. Bot. 68, 4869-4884 (2017).

4. Ku, H. M., Doganlar, S., Chen, K. Y. \& Tanksley, S. D. The genetic basis of pearshaped tomato fruit. Theor. Appl. Genet. 99, 844-850 (1999).

5. Liu, J., Van Eck, J., Cong, B. \& Tanksley, S. D. A new class of regulatory genes underlying the cause of pear-shaped tomato fruit. Proc. Natl Acad. Sci. USA 99, 13302-13306 (2002)

6. Hackbusch, J., Richter, K., Müller, J., Salamini, F. \& Uhrig, J. F. A central role of Arabidopsis thaliana ovate family proteins in networking and subcellular localization of 3-aa loop extension homeodomain proteins. Proc. Natl Acad. Sci. USA 102, 4908-4912 (2005).

7. Wang, S., Chang, Y., Guo, J. \& Chen, J. G. Arabidopsis Ovate Family Protein 1 is a transcriptional repressor that suppresses cell elongation. Plant J. 50, 858-872 (2007).

8. Wang, Y. K. et al. Ovate family protein 1 as a plant Ku70 interacting protein involving in DNA double-strand break repair. Plant Mol. Biol. 74, 453-466 (2010).

9. Wang, S. et al. Arabidopsis Ovate family proteins, a novel transcriptional repressor family, control multiple aspects of plant growth and development. PloS ONE 6, https://doi.org/10.1371/journal.pone.0023896 (2011).

10. Li, E., Wang, S., Liu, Y., Chen, J. G. \& Douglas, C. J. OVATE FAMILY PROTEIN4 (OFP4) interaction with KNAT7 regulates secondary cell wall formation in Arabidopsis thaliana. Plant J. 67, 328-341 (2011).

11. Zhang, L. et al. Ovate family protein1 interaction with $\mathrm{BLH} 3$ regulates transition timing from vegetative to reproductive phase in Arabidopsis. Biochem. Biophys. Res. Commun. 470, 492-497 (2016).

12. Schmitz, A. J., Begcy, K., Sarath, G. \& Walia, H. Rice Ovate Family Protein 2 (OFP2) alters hormonal homeostasis and vasculature development. Plant Sci. 241, 177-188 (2015)
13. Yang, C., Shen, W., He, Y., Tian, Z. \& Li, J. OVATE family protein 8 positively mediates brassinosteroid signaling through interacting with the GSK3-like kinase in rice. PLoS Genet. 12, https://doi.org/10.1371/journal.pgen.1006118 (2016).

14. Xiao, Y., Liu, D., Zhang, G., Tong, H. \& Chu, C. Brassinosteroids regulate OFP1, a DLT interacting protein, to modulate plant architecture and grain morphology in rice. Front. Plant Sci. 8, 1698 (2017).

15. Yang, C., Ma, Y., He, Y., Tian, Z. \& Li, J. OsOFP19 modulates plant architecture by integrating the cell division pattern and brassinosteroid signaling. Plant J. 93, 489-501 (2018).

16. Liu, J. et al. Banana ovate family protein MaOFP1 and MADS-box protein MUMADS1 antagonistically regulated banana fruit ripening. PLOS ONE 10, e0123870 (2015).

17. Liu, J. et al. MuMADS1 and MaOFP1 regulate fruit quality in a tomato ovate mutant. Plant Biotechnol. J. 16, 989-1001 (2018).

18. Wang, S., Chang, Y. \& Ellis, B. Overview of OVATE FAMILY PROTEINS, A novel class of plant-specific growth regulators. Front. Plant Sci. 7, 417 (2016).

19. Pagnussat, G. C., Yu, H. J. \& Sundaresan, V. Cell-fate switch of synergid to egg cell in Arabidopsis eostre mutant embryo sacs arises from misexpression of the BEL1-like homeodomain gene BLH1. Plant Cell 19, 3578-3592 (2007).

20. Gong, S. Y. et al. Cotton KNL1, encoding a class II KNOX transcription factor, is involved in regulation of fibre development. J. Exp. Bot. 65, 4133-4147 (2014).

21. Depuydt, S. \& Hardtke, C. S. Hormone signalling crosstalk in plant growth regulation. Curr. Biol. 21, R365-R373 (2011).

22. Klee, H. J. \& Giovannoni, J. J. Genetics and control of tomato fruit ripening and quality attributes. Annu. Rev. Genet. 45, 41-59 (2011).

23. Kimura, S. \& Sinha, N. Tomato (Solanum lycopersicum): a model fruit-bearing crop. CSH Protoc. 2008, pdb emo105 (2008).

24. Huang, Z., Houten, J. V., Gonzalez, G., Han, X. \& Knaap, E. V. D. Genome-wide identification, phylogeny and expression analysis of SUN, OFP and YABBY gene family in tomato. Mol. Genet. Genomics 288, 111-129 (2013).

25. Wu, S. et al. A common genetic mechanism underlies morphological diversity in fruits and other plant organs. Nat. Commun. 9, 4734 (2018).

26. Zhu, $M$. et al. A new tomato NAC (NAM/ATAF1/2/CUC2) transcription factor, SINAC4, functions as a positive regulator of fruit ripening and carotenoid accumulation. Plant Cell Physiol. 55, 119-135 (2014).

27. Chen, $\mathrm{G}$. et al. Identification of a specific isoform of tomato lipoxygenase (TomloxC) involved in the generation of fatty acid-derived flavor compounds. Plant Physiol. 136, 2641-2651 (2004).

28. Zhou, S. et al. Manipulation of plant architecture and fl owering time by down-regulation of the GRAS transcription factor SIGRAS26 in Solanum lycopersicum. Plant Sci. 271, 81-93 (2018).

29. Expósito-Rodríguez, M., Borges, A. A., Borges-Pérez, A. \& Pérez, J. A. Selection of internal control genes for quantitative real-time RT-PCR studies during tomato development process. BMC Plant Biol. 8, 131 (2008).

30. $\mathrm{Yu}, \mathrm{X}$. et al. The Jasmonate ZIM-domain protein gene SIJAZ2 regulates plant morphology and accelerates flower initiation in Solanum lycopersicum plants. Plant Sci. 267, 65-73 (2018).

31. Fragkostefanakis, S. et al. HsfA2 controls the activity of developmentally and stress-regulated heat stress protection mechanisms in tomato male reproductive tissues. Plant Physiol. 170, 2461-2477 (2016).

32. Huang, W. et al. Overexpression of a tomato miR171 target gene SIGRAS24 impacts multiple agronomical traits via regulating gibberellin and auxin homeostasis. Plant Biotechnol. J. 15, 472-488 (2017).

33. Guo, X. et al. The tomato floral homeotic protein FBP1-like gene, SIGLO1, plays key roles in petal and stamen development. Sci. Rep. 6, 20454 (2016).

34. Huang, W., Xian, Z., Kang, X., Tang, N. \& Li, Z. Genome-wide identification, phylogeny and expression analysis of GRAS gene family in tomato. BMC Plant Biol. 15, 209 (2015).

35. Noguchi, T. et al. Brassinosteroid-insensitive dwarf mutants of Arabidopsis accumulate brassinosteroids. Plant Physiol. 121, 743-752 (1999).

36. Xiao, J. et al. Dissection of GA 20-oxidase members affecting tomato morphology by RNAi-mediated silencing. Plant Growth Regul. 50, 179-189 (2006).

37. Yamaguchi, S. Gibberellin metabolism and its regulation. J. Plant Growth Regul. 20, 317-318 (2001).

38. Hedden, P. \& Kamiya, Y. GIBBERELLIN BIOSYNTHESIS: enzymes, genes and their regulation. Annu. Rev. Plant Physiol. Plant Mol. Biol. 48, 431-460 (1997).

39. Serrani, J. C., Ruiz-Rivero, O., Fos, M. \& Garcia-Martinez, J. L. Auxin-induced fruitset in tomato is mediated in part by gibberellins. Plant J. 56, 922-934 (2008). 
40. Zhang, L. Y. et al. Antagonistic $\mathrm{HLH} / \mathrm{bHLH}$ transcription factors mediate brassinosteroid regulation of cell elongation and plant development in rice and Arabidopsis. Plant Cell 21, 3767-3780 (2009).

41. Lee, S. et al. Overexpression of PRE1 and its homologous genes activates Gibberellin-dependent responses in Arabidopsis thaliana. Plant Cell Physiol. 47, 591-600 (2006).

42. Pnueli, L., Hareven, D., Rounsley, S. D., Yanofsky, M. F. \& Lifschitz, E. Isolation of the tomato AGAMOUS gene TAG1 and analysis of its homeotic role in transgenic plants. Plant Cell 6, 163-173 (1994).

43. Pnueli, L., Hareven, D., Broday, L., Hurwitz, C. \& Lifschitz, E. The TM5 MADS box gene mediates organ differentiation in the three inner whorls of tomato flowers. Plant Cell 6, 175-186 (1994).

44. Ampomahdwamena, C., Morris, B. A., Sutherland, P., Veit, B. \& Yao, J. L. Downregulation of TM29, a TomatoSEPALLATA homolog, causes parthenocarpic fruit development and floral reversion. Plant Physiol. 130, 605-617 (2002).

45. Kim, W. B. et al. Identification of a pollen-specific gene, SICRK1 (RFK2) in tomato. Genes Genom. 36, 303-311 (2014).

46. Kim, W. B. et al. SIPMEl, a pollen-specific gene in tomato. Can. J. Plant Sci. 94 73-83 (2017).

47. Covey, P. A. et al. A pollen-specific RALF from tomato that regulates pollen tube elongation. Plant Physiol. 153, 703-715 (2010).

48. Kim, H. U. et al. New pollen-specific receptor kinases identified in tomato, maize and Arabidopsis: the tomato kinases show overlapping but distinct localization patterns on pollen tubes. Plant Mol. Biol. 50, 1-16 (2002).

49. Tang, W., Ezcurra, l., Muschietti, J. \& McCormick, S. A cysteine-rich extracellular protein, LAT52, interacts with the extracellular domain of the pollen receptor kinase LePRK2. Plant Cell 14, 2277 (2002).

50. Annadana, S. et al. Cloning of the chrysanthemum UEP1 promoter and comparative expression in florets and leaves of Dendranthema grandiflora. Transgenic Res. Transgenic Res. 11, 437 (2002).

51. Faktor, O., Kooter, J. M., Dixon, R. A. \& Lamb, C. J. Functional dissection of a bean chalcone synthase gene promoter in transgenic tobacco plants reveals sequence motifs essential for floral expression. Plant Mol. Biol. 32, 849-859 (1996)

52. Yu, D. \& Chen, Z. Evidence for an important role of WRKY DNA binding proteins in the regulation of NPR1 gene expression. Plant Cell 13, 1527-1540 (2001).

53. Rogers, H. J. et al. Functional analysis of cis-regulatory elements within the promoter of the tobacco late pollen gene g10. Plant Mol. Biol. 45, 577-585 (2001).

54. Xia, X. J. et al. Interplay between reactive oxygen species and hormones in the control of plant development and stress tolerance. J. Exp. Bot. 66, 2839-2856 (2015).

55. Wang, Z. Y., Zhu, J. Y. \& Sae-Seaw, J. Brassinosteroid signaling. Development 140, 1615-1620 (2013).
56. She, J. et al. Structural insight into brassinosteroid perception by BRI1. Nature 474, 472-477 (2011).

57. Hothorn, M. et al. Structural basis of steroid hormone perception by the receptor kinase BRI1. Nature 474, 467-472 (2011).

58. Li, J. \& Chory, J. A putative leucine-rich repeat receptor kinase involved in brassinosteroid signal transduction. Cell 90, 929-936 (1997).

59. Yin, Y. et al. A new class of transcription factors mediates brassinosteroid-regulated gene expression in Arabidopsis. Cell 120, 249-259 (2005).

60. He, J. X. et al. BZR1 is a transcriptional repressor with dual roles in brassinosteroid homeostasis and growth responses. Science 307, 1634-1638 (2005).

61. Sato, Y. et al. Abnormal cell divisions in leaf primordia caused by the expression of the rice homeobox geneOSH1 lead to altered morphology of leaves in transgenic tobacco. Mol. Gen. Genet. 251, 13-22 (1996).

62. Tong, $\mathrm{H}$. et al. DWARF and LOW-TILLERING, a new member of the GRAS family, plays positive roles in brassinosteroid signaling in rice. Plant J. 58, 803-816 (2009)

63. Tsuda, K., Kurata, N., Ohyanagi, H. \& Hake, S. Genome-wide study of KNOX regulatory network reveals brassinosteroid catabolic genes important for shoot meristem function in rice. Plant Cell 26, 3488-3500 (2014).

64. Chory, J., Nagpal, P. \& Peto, C. A. Phenotypic and genetic analysis of det2, a new mutant that affects light-regulated seedling development in Arabidopsis. Plant Cell 3, 445-459 (1991).

65. Stewart Lilley, J. L., Gan, Y., Graham, I. A. \& Nemhauser, J. L. The effects of DELLAs on growth change with developmental stage and brassinosteroid levels. Plant J. 76, 165-173 (2013).

66. Tong, $\mathrm{H}$. et al. Brassinosteroid regulates cell elongation by modulating gibberellin metabolism in rice. Plant Cell 26, 4376-4393 (2014)

67. Zhu, Z. et al. Overexpression of SIPRE2, an atypical bHLH transcription factor affects plant morphology and fruit pigment accumulation in tomato. Sci. Rep. 7, 5786 (2017).

68. Ruan, Y. L., Patrick, J. W., Bouzayen, M., Osorio, S. \& Fernie A. R. Molecular regulation of seed and fruit set. Trends Plant Sci. 17, 656-665 (2012).

69. Kim, T. W. et al. Arabidopsis CYP85A2, a cytochrome P450, mediates the Baeyer-Villiger oxidation of castasterone to brassinolide in brassinosteroid biosynthesis. Plant Cell 17, 2397-2412 (2005).

70. Szekeres, M. et al. Brassinosteroids rescue the deficiency of CYP90, a cytochrome P450, controlling cell elongation and de-etiolation in Arabidopsis. Cell 85, 171-182 (1996).

71. Ye, Q. et al. Brassinosteroids control male fertility by regulating the expression of key genes involved in Arabidopsis anther and pollen development. Proc. Natl Acad. Sci. USA 107, 6100-6105 (2010). 